\title{
DETERMINACIÓN INVERSA DE LA CONDUCTIVIDAD TERMICA EN MADERA DE Pinus radiata
}

\section{Inverse determination of thermal conductivity in wood of Pinus radiata}

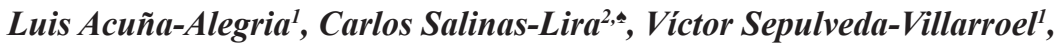 \\ Diego Vasco-Calle ${ }^{3}$ Rubén A. Ananías ${ }^{1}$
}

\begin{abstract}
RESUMEN
Se realizan experimentos a escala de laboratorio para caracterizar el secado artificial de madera de pino radiata a temperaturas convencionales y aceleradas, con el propósito de obtener datos que permitan determinar la conductividad térmica de la madera mediante metodología inversa. El análisis comparativo muestra que la implementación de la metodología inversa desarrollada entrega información consistente con datos disponibles en la literatura especializada: conductividades térmicas crecientes con el aumento de la humedad y la temperatura, cuyos valores fluctúan entre 0,34 y $0,56(\mathrm{~W} / \mathrm{m} \mathrm{K})$ para variaciones de humedad entre $22 \%$ y $64 \%$; y temperaturas entre $70^{\circ} \mathrm{C}$ a $90^{\circ} \mathrm{C}$, respectivamente.
\end{abstract}

Palabras Claves: Calor específico, coeficiente de transferencia de calor, curvas de secado, propiedades térmicas, secado de madera.

\begin{abstract}
Laboratory scale experiments are performed in order to characterize the artificial wood drying of Pinus radiata at conventional temperatures and accelerated temperatures. The experimental data allow determining the wood thermal conductivity through inverse methodology. The comparative analysis shows that the implementation of the developed inverse methodology is consistent with information available in the specialized literature; which means increased thermal conductivities with increased humidity and temperature, whose values oscillate between 0,34 and $0,56(\mathrm{~W} / \mathrm{m} \mathrm{K})$ to moisture variations between $22 \%$ and $64 \%$; and temperatures between $70^{\circ} \mathrm{C}$ and $90^{\circ} \mathrm{C}$, respectively.
\end{abstract}

Keywords: Drying curves, heat transfer coefficient, inverse problem, radiata pine, specific heat, thermal properties, wood drying.

\footnotetext{
${ }^{1}$ Grupo de Investigación en Tecnologías del Secado y Tratamientos Térmicos de la Madera. Departamento de Ingeniería en Maderas, Universidad del Bío-Bío, Chile.

${ }^{2}$ Grupo de Investigación en Tecnologías del Secado y Tratamientos Térmicos de la Madera. Departamento de Ingeniería Mecánica, Universidad del Bío-Bío, Chile.

${ }^{3}$ Grupo de Investigación en Tecnologías del Secado y Tratamientos Térmicos de la Madera. Departamento de Ingeniería en Mecánica, Universidad de Santiago de Chile, Chile.

•Corresponding author: casali@ubiobio.cl
}

Received: 16.04.2017 Accepted: 04.05.2018 


\section{INTRODUCCIÓN}

La madera de pino radiata representa el $96 \%$ de la industria nacional del aserrío, con una producción anual de 8,3 millones de metros cúbicos (INFOR 2016). Esta especie, particularmente su albura, al presentar una configuración leñosa de alta permeabilidad, permite el uso de diversos programas de secado caracterizados por su temperatura bulbo seco y húmedo $\left(\mathrm{T}_{\mathrm{BS}} / \mathrm{T}_{\mathrm{BH}}\right)$ : $70 / 50^{\circ} \mathrm{C}-140 / 90^{\circ} \mathrm{C}$ (Ananías et al. 2005). No obstante, sus propiedades varían fuertemente en su interior producto de las características anatómicas que la gobiernan (Díaz-Vaz et al. 2007, Ananías et al. 2010). En este contexto, la pared celular no es uniforme, y en consecuencia, induce variaciones en las propiedades físicas y mecánicas de la madera, entre ellas, la conductividad térmica.

La conductividad térmica $(\mathrm{k})$ depende del material y su configuración. En consecuencia, considera la composición celular heterogénea de la madera: forma, dimensión, orientación, composición química y ultra-estructura y ciertamente, su contenido de humedad (CH) (Díaz-Vaz et al. 2009, Pérez-Peña 2011). Dentro de la diversidad de factores que afectan la conductividad térmica en la madera, diversos autores han manifestado que las propiedades que más influyen en la magnitud de $\mathrm{k}$ son el $\mathrm{CH}$ y la densidad de la madera (Cai y Garrahan 2006, Haque 2007).

En particular, en el secado de madera, se emplean modelos matemáticos que se encargan de describir adecuadamente fenómenos de transporte de calor y masa basado en sistemas de ecuaciones diferenciales. Dichos modelos pueden ser agrupados en tres tipos: modelos fenomenológicos (Salinas et al. 2008), según enfoque de Luikov (1966) y multi-fásicos (Whittaker 1977), una revisión de este tema es dada por (Zhang et al. 2014). Particularmente relevante resultan los trabajos que incorporan etapas de secado ampliamente documentadas en la literatura relacionadas con el contenido de humedad crítico (CHC) y el punto de saturación de la fibra (PSF) (Derdour et al. 2007 and Zhao et al. 2016). Derdour et al. 2007 determinan experimentalmente el contenido de humedad crítico para varias condiciones de secado y Zhao et al. (2016), desarrollan un modelo que contempla una interfase variable asociada al desplazamiento del frente de evaporación.

La solución de modelos matemáticos diferenciales requiere de métodos numéricos de integración y por cierto, del cálculo computacional. El Método de Volúmenes Finitos (MVF), es una herramienta numérica para la integración de modelos matemáticos diferenciales, que consiste en subdividir un cierto dominio de cálculo en un número finito de volúmenes de control, cuyo centroide (nodo) es representado por el valor medio de la variable dependiente. A continuación, se integra la ecuación diferencial gobernante en cada volumen de control, evaluando los diferenciales en forma discreta (Patankar 1980). De lo anterior resulta un sistema de ecuaciones algebraicas, de cuya solución se obtienen los valores nodales de la variable dependiente (Versteeg y Malalasekera 1995). El método ha sido ampliamente utilizado y reconocido por la comunidad del Cálculo Computacional de Dinámica de Fluidos, particularmente por ser intrínsecamente conservativo (Salinas et al. 2015).

Normalmente, los problemas de la física que derivan en modelos matemáticos diferenciales, tienen variables dependientes, la cuales adquieren un valor en un determinado dominio de cálculo (variables independientes), teniendo como parámetros las propiedades físicas del dominio y condicionantes de valor inicial y de contorno. De este contexto derivan los denominados problemas inversos, es decir: dada la distribución espacial de las variables dependientes determinar parámetros físicos y/o condiciones iniciales y de contorno de dichas distribuciones. Por ejemplo, en transferencia de calor, el problema inverso puede estar asociado a la estimación de un flujo de calor desconocido en el borde de un material, usando mediciones de temperatura en las cercanías de la superficie (Özisik y Orlande 2000). La principal ventaja de este método es la mayor afinidad de resultados productos de investigaciones experimentales y teóricas, sumado al hecho, que no siempre es factible por métodos directos, determinar las causas (conductividades) para conocer los efectos (temperaturas) debido a limitaciones técnicas o físicas.

En el contexto de la madera, dicha técnica fue aplicada por Liu et al. (2001) para estimar el coeficiente difusivo en madera de Quercus rubra bajo condiciones de secado de $43,4 / 28,5\left({ }^{\circ} \mathrm{C} /{ }^{\circ} \mathrm{C}\right)$. El análisis numérico empleado para determinar la distribución espacial y temporal del coeficiente difusivo fue el Método de Diferencias Finitas (MDF). El resultado final permitió concluir que el coeficiente de 
difusión es función únicamente del $\mathrm{CH}$, aumentando rápidamente desde el $\mathrm{CH}$ de Equilibrio (CHE) $(5,5 \%)$ hasta un $15 \%$ aproximadamente, posterior a lo cual, el incremento se desacelera de manera gradual hasta alcanzar el PSF. Además, Cai (2005) utilizó el método inverso para determinar el coeficiente de difusión de $\mathrm{CH}$ en el secado de madera de Abies lasiocarpa, utilizando datos de distribución de $\mathrm{CH}$ de un programa de secado $60 / 47,5\left({ }^{\circ} \mathrm{C}\right)$. Los resultados fueron comparados con el método tradicional de cálculo. Finalmente se determinó que ambos resultados eran similares y se pudo concluir que las dos técnicas empleadas eran adecuadas para el cálculo de parámetros relacionados con el secado de madera. En particular, Cai y Garrahan (2006), desarrollaron un algoritmo inverso utilizando el MDF, que permite la determinación de la distribución espacial y temporal de la conductividad térmica entre el centro y la superficie en madera de Tsuga heterophyla, utilizando temperaturas de $70 / 70\left({ }^{\circ} \mathrm{C} /{ }^{\circ} \mathrm{C}\right)$. Se concluye en la investigación que la conductividad térmica es un parámetro dependiente del $\mathrm{CH}$, por lo que disminuye a medida que la madera se seca y también varía en el intervalo centro/superficie debido al gradiente de humedad producido durante el proceso. Igualmente, Czajkowski et al. (2016), lo ha aplicado para determinar propiedades térmicas en paneles de madera. En adición, los métodos inversos pueden darse en un contexto de búsqueda extensiva de parámetros físicos de interés. En este contexto el trabajo Zheng et al. (2016), lo realiza con la difundida técnica de algoritmos genéticos para determinar coeficientes de difusión efectiva.

En este contexto se desarrolla un procedimiento basado en un modelo matemático diferencial, integrado numéricamente a través del MVF, que permite la determinación de la conductividad térmica mediante metodología inversa y su validación, aplicado a tres programas de secado: $70 / 50\left({ }^{\circ} \mathrm{C}\right)$; $80 / 55\left({ }^{\circ} \mathrm{C}\right)$ y $90 / 60\left({ }^{\circ} \mathrm{C}\right)$. La principal característica del método implementado es que no se requiere información previa sobre la dependencia de la conductividad del $\mathrm{CH}$, además de resultar en un sistema ecuaciones lineales resuelto por regresión sucesiva.

\section{MATERIALES Y MÉTODOS}

En el presente estudio se utilizó madera lateral de pino radiata (Pinus radiata D. Don) proveniente de plantaciones forestales del fundo La Candelaria (Sector El Venado), San Pedro de la Paz, Región del Biobío, Chile. El árbol seleccionado estaba libre de defectos, tenía 14 años y un diámetro a la altura del pecho (DAP) igual $39(\mathrm{~cm})$.

Se contempla la realización de cuatro experimentos que permiten generar una base de datos para determinar y validar diversos parámetros físicos presentes en modelos matemáticos diferenciales del proceso de secado. En particular, determinar la conductividad térmica de madera de pino radiata como función de su $\mathrm{CH}$ a través de metodología inversa, usando distribuciones transitorias y espaciales de $\mathrm{CH}$ y temperatura en tres condiciones de secado (convencional y acelerado): 70/50, 80/55 y 90/60 $\left({ }^{\circ} \mathrm{C}\right.$ ).

Para efectos de propiciar un flujo unidimensional de calor y $\mathrm{CH}$ se utilizaron probetas de $30 \times 5 \times 4$ $(\mathrm{cm})$ dispuestas en un panel de madera de 48x30x4 $(\mathrm{cm})$, conformado por cuatro tablas prensadas y selladas (Figura 1). Las probetas de corte radial (a) y tangencial (b) son obtenidas de la tabla A y B, respectivamente. Dichas probetas sirven al propósito de evaluar distribuciones temporales y espaciales de $\mathrm{CH}$ y temperatura. 


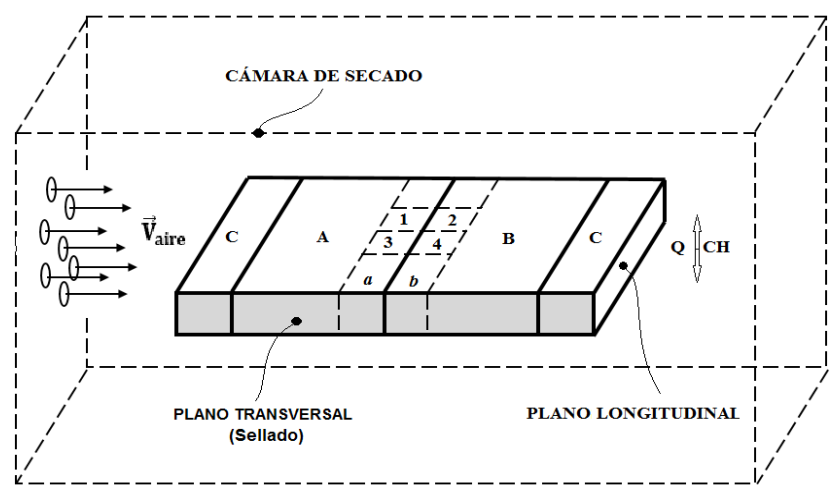

Figura 1: Panel de madera de 48x30x4 (cm): Tabla de corte radial y tangencial (A y B); Tabla complementaria (C); Probeta de 30x5 4 ( $\mathrm{cm}$ ) de corte radial (a) y tangencial (b); Muestras de 4x5 4 $(\mathrm{cm})$ de corte radial (1 y 3$)$ y tangencial (2 y 4) para evaluación de distribución espacial de humedad.

\begin{tabular}{|c|c|c|c|c|c|}
\hline \multicolumn{7}{|c|}{ Tabla 1: Diseño Experimental. } \\
\hline \multirow{2}{*}{ Ensayo } & \multicolumn{2}{|c|}{ Sentido del Flujo } & \multicolumn{3}{c|}{ Programa de Secado } \\
\cline { 2 - 6 } & Radial & Tangencial & $70 / 50\left({ }^{\circ} \mathrm{C}\right)$ & $80 / 55\left({ }^{\circ} \mathrm{C}\right)$ & $90 / 60\left({ }^{\circ} \mathrm{C}\right)$ \\
\hline 1 & $\mathbf{x}$ & & $\mathbf{x}$ & & \\
\hline 2 & & $\mathbf{x}$ & $\mathbf{x}$ & & \\
\hline 3 & $\mathbf{x}$ & & & $\mathbf{x}$ & \\
\hline 4 & & $\mathbf{x}$ & & & $\mathbf{x}$ \\
\hline 5 & $\mathbf{x}$ & & & & $\mathbf{x}$ \\
\hline 6 & & $\mathbf{x}$ & & & \\
\hline
\end{tabular}

\section{Curvas de secado}

Se efectuaron dos ensayos por cada programa de secado (Tabla 1) y sentido de flujo (radial y tangencial). Debido a que las evaluaciones son de carácter destructivo, se prestó especial atención a la uniformidad de las probetas en cuanto al tipo de madera: madera lateral con similar proporción de madera temprana y tardía. Los ensayos contemplan una réplica, en consecuencia, se realizaron un total de 12 ensayos (6 radiales y 6 tangenciales).

Se utilizaron probetas de 4x5x30 (cm) para evaluación, de acuerdo con la norma chilena NCh 968 . Of 86, dispuestas en un panel de madera, conforme descrito en la sección precedente. A dichas probetas (Figura 2) se les controló su masa cada cierto tiempo Dt, variable conforme la etapa de secado $(0,2 \mathrm{~h}$ $<\mathrm{Dt}<10 \mathrm{~h}$,), para determinar su $\mathrm{CH}$ y curva de secado.

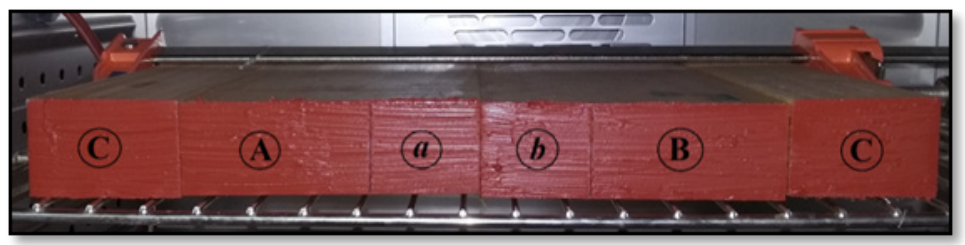

Figura 2: Probetas a y b dispuestas en el panel para efectos de determinar curvas de secado.

\section{Distribuciones de temperatura}

Se determinaron temperaturas transitorias en cuatro puntos equidistantes entre el centro y la superficie de la madera, para flujos de humedad unidimensional en el sentido radial y tangencial (Figura 3). Se registró cada 2 minutos la señal de los termopares con un equipo marca FLUKE modelo Hydra II, monitoreados por un computador. Las evaluaciones contemplaron tres programas de secado, 
dos direcciones de flujo y una réplica para cada condición, de lo cual resultó un total de 12 evaluaciones (6 radiales y 6 tangenciales). Los termopares fueron instalados en forma transversal en dos probetas de $30 \times 5 \times 4 \mathrm{~cm}$, cuidando de obtener una efectiva localización y contacto del sensor: profundidad requerida, necesario aislamiento y libre de espacios vacíos inducidos por la perforación de $1,5 \mathrm{~mm}$ de diámetro (Figura 3a). Las probetas se dispusieron en la parte central de un panel de madera $(48 \times 30 \times 4$ $\mathrm{cm}$ ), compuesto de tablas prensadas y selladas (Figura 3b).

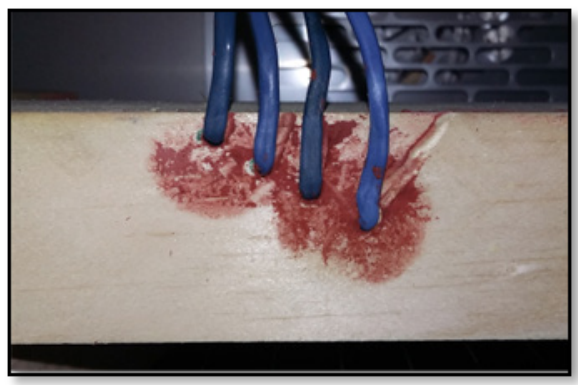

a

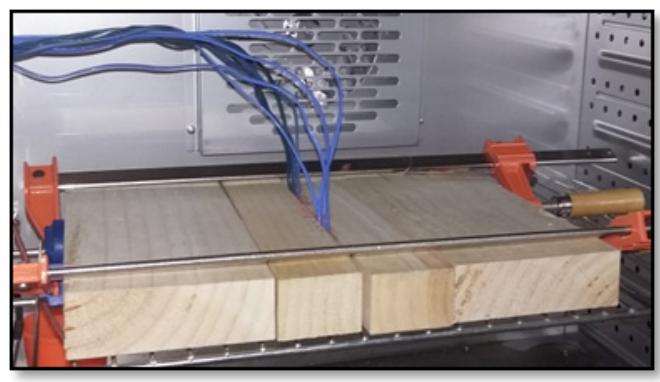

b

Figura 3: Sensores de temperaturas: a) detalle de instalación de termopares. b) montaje al interior de la cámara: termopares instalados, previo al prensado y sellado.

\section{Distribuciones de contenido de humedad}

Se determinaron distribuciones espaciales unidimensionales de $\mathrm{CH}$ en 8 posiciones (Figura 4), distribuidas equidistantes entre el centro y la superficie de la madera, en el sentido de flujo de humedad radial y tangencial, para cinco tiempos de secado (Figura 8). Este experimento se replicó una vez, por lo que se necesitó un total de 60 probetas (30 radiales y 30 tangenciales). En este caso, se asumió simetría en el sentido de flujo, por lo cual sólo se seccionó la mitad superior de la probeta (Figura 4b).

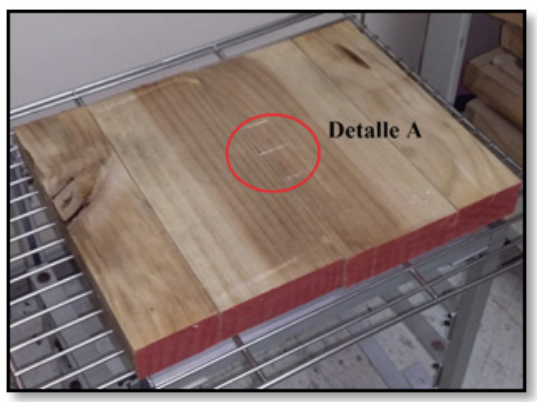

a

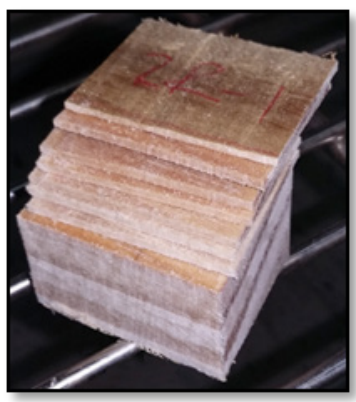

b

Figura 4: Panel compuesto: a) El "Detalle A" indica la posición de las muestras destinadas a la medición espacial de humedad, b) Muestra seccionada entre el centro y la superficie de la madera.

\section{Medición de propiedades térmicas}

Para efectos de contrastar valores de conductividad térmica obtenidos por métodos tradicionales versus los determinados con metodología inversa, además del valor de calor específico requerido por el modelo matemático, se implementaron evaluaciones experimentales de conductividad térmica y calor específico, utilizando el instrumento resistivo Decagon KD2-Pro, en dos direcciones principales 
de la madera: radial y tangencial, a diversos $\mathrm{CH}$ y a $70{ }^{\circ} \mathrm{C}$. Se contemplan dos réplicas, lo cual implica el uso de 6 probetas de 40x40x40 (mm). En este contexto, las probetas requieren dos perforaciones (1,3 $\mathrm{mm}$ de diámetro, $30 \mathrm{~mm}$ de profundidad y $6 \mathrm{~mm}$ de separación) para la instalación del sensor del instrumento Decagon KD2-Pro (Figura 5a). Lo anterior fue realizado conforme las recomendaciones del fabricante en cuanto a las dimensiones mínimas de la probeta y a la instalación ajustada del sensor, previamente tratado con pasta térmica para minimizar los efectos de resistencia de contacto.

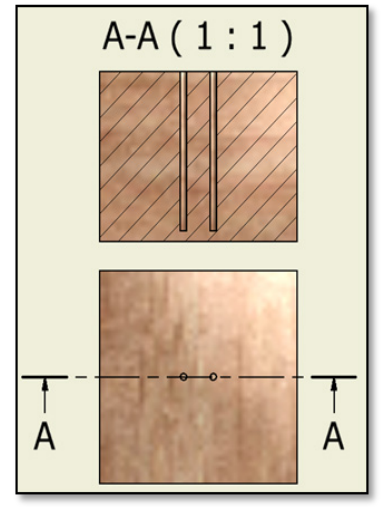

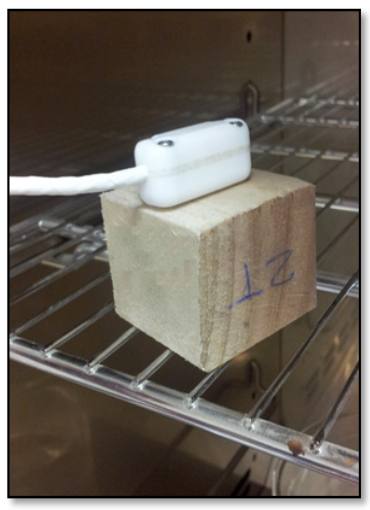

b

Figura 5: Probetas para determinación de conductividad térmica y calor específico: a) perforaciones, b) instalación.

\section{Modelo matemático}

El calor que fluye a través de una pieza de madera en dirección del espesor $L$, puede representarse mediante una ecuación diferencial parcial no lineal de segundo orden, mediante del siguiente modelo unidimensional:

$$
\rho c_{p}(C H) \frac{\partial T}{\partial t}=\frac{\partial}{\partial x}\left(\lambda(C H) \frac{\partial T}{\partial x}\right) \quad 0<x<L, \quad t>0
$$

Donde $\rho$ es la densidad de la madera $\left(\mathrm{kg} / \mathrm{m}^{3}\right) ; C_{p}(\mathrm{CH})$ es el calor específico de la madera $(\mathrm{J} / \mathrm{kg} \mathrm{K})$ en función del $\mathrm{CH} ; T$ es la temperatura $(\mathrm{K}) ; \mathrm{t}$ es el tiempo (s); $\lambda(\mathrm{CH})$ es la conductividad térmica $(\mathrm{W} / \mathrm{m} \mathrm{K})$ en función del $\mathrm{CH}$; y $x$ es la coordenada espacial medida desde el centro hacia la superficie de la probeta.

Condicionada por:

$$
\left.\begin{array}{c}
\left.\lambda(\mathrm{CH}) \frac{\partial T}{\partial x}\right|_{x=L}=\alpha(\mathrm{CH})\left(T_{\infty}-T_{s}(\mathrm{CH})\right) \\
\left.\frac{\partial T}{\partial x}\right|_{x=0}=0
\end{array}\right\}
$$

$\left.T(x)=T_{0}\right\}$ valor inicial 
Donde $\mathrm{T}_{\mathrm{s}}(\mathrm{CH})$ es la temperatura en la superficie de la madera $(\mathrm{K})$ en función del $\mathrm{CH}, T_{\infty}$ es la temperatura del ambiente de secado $(\mathrm{K})$ y $\alpha(\mathrm{CH})$ es el coeficiente convectivo de transferencia de calor $\left(\mathrm{W} / \mathrm{m}^{2} \mathrm{~K}\right)$ en función del $\mathrm{CH}$.

Para efectos de integración numérica de (1), basado en el MVF, se consideran $N$ volúmenes finitos en el dominio $0<\mathrm{x}<\mathrm{L}$, donde el centroide es representado por el valor medio de la variable distinguido por el subíndice $P\left(\mathrm{~T}_{\mathrm{p}}\right)$ y los valores de $\mathrm{T}$ en los volúmenes adyacentes: $\mathrm{T}_{\mathrm{w}}$ (atrás) y $\mathrm{T}_{\mathrm{E}}$ (adelante). Los límites del volumen finito centrado en $P$ se identifican como $w$ y $e$, respectivamente (Figura 6 ).

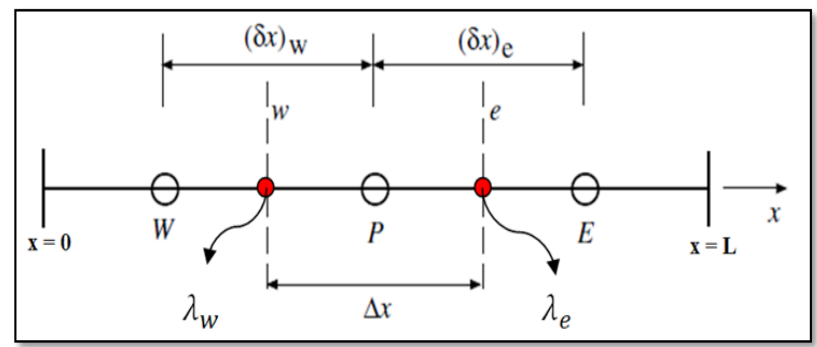

Figura 6. Subdivisión de dominios según el Método de Volúmenes Finitos.

En este sentido, la expresión para el volumen finito centrado en $\mathrm{P}$, evaluada implícitamente en el tiempo $t=k \Delta \mathrm{t} y \mathrm{t}=(\mathrm{k}+1) \Delta \mathrm{t}$ es:

$$
\rho c_{p} \frac{\Delta x}{\Delta t}\left(T_{p}^{k+1}-T_{p}^{k}\right)=\frac{\lambda_{e}\left(T_{E}^{k+1}-T_{p}^{k+1}\right)}{(\delta x)_{e}}-\frac{\lambda_{w}\left(T_{p}^{k+1}-T_{w}^{k+1}\right)}{(\delta x)_{w}}
$$

Ahora, definiendo como variable dependiente $\lambda$, se puede escribir:

$$
\lambda_{w} * a_{w}+\lambda_{e} * a_{e}=b
$$

Siendo $a_{w}=-\frac{\left(T_{p}^{k+1}-T_{w}^{k+1}\right)}{(\delta x)_{w}} ; a_{e}=-\frac{\left(T_{E}^{k+1}-T_{p}^{k+1}\right)}{(\delta x)_{e}} ; b=\rho c_{p} \frac{\Delta x}{\Delta t}\left(T_{p}^{k+1}-T_{p}^{k}\right)$

Si $i=1, N-1$ denota al VF, siendo $N$ el número de VF. Entonces se pueden escribir $N-1$ ecuaciones genéricas del tipo (3), quedando:

$$
\lambda_{i} * a_{w_{i}}+\lambda_{i+1} * a_{e_{i}}=b_{i}
$$

Siendo $T_{w}=T_{i-1} ; T_{p}=T_{i} ; T_{E}=T_{i+1}$

Luego, la ecuación para el VF en $i=N$, requiere incorporar la condición de contorno de (1) en $\mathrm{x}=\mathrm{L}$ (contorno expuesto a convección). Por lo tanto, se tiene: 


$$
\rho c_{p} \frac{\Delta x}{\Delta t}\left(T_{p}^{k+1}-T_{p}^{k}\right)=\left.\frac{\lambda_{e}\left(T_{E}^{k+1}-T_{P}^{k+1}\right)}{(\delta x)_{e}}\right|_{q=a\left(T_{\infty}-T_{s}\right)}-\frac{\lambda_{w}\left(T_{p}^{k+1}-T_{w}^{k+1}\right)}{(\delta x)_{w}}
$$

Modelado por extrapolación lineal el valor de $\mathrm{T}_{\mathrm{s}}$, se tiene la siguiente ecuación para el VF i=N:

$$
\lambda_{n} * q_{w_{n}}=b_{n}
$$

Siendo $a_{w_{n}}=-\frac{\left(\mathrm{T}_{n}^{k+1}-\mathrm{T}_{n-1}^{k+1}\right)}{(\delta x) w_{n}}$

$$
b_{n}=\rho c_{p} \frac{\Delta x}{\Delta t}\left(T_{n}^{k+1}-T_{n}^{k}\right)-\alpha\left[T_{\infty}-T_{n-1}^{k+1}+\left(T_{n-1}^{k+1}-T_{n}^{k+1}\right)\left(1+\frac{\Delta x_{n}}{2(\delta x) w_{n}}\right)\right]
$$

Luego, si $\lambda_{i}=\lambda_{w} \quad$ y $\lambda_{i+1}=\lambda_{e} \quad(\mathrm{i}=1, \mathrm{~N})$, se puede escribir:

$$
A \lambda=b
$$

Donde $\boldsymbol{A}$ es la matriz de coeficientes, del tipo bi-diagonal superior, $\lambda$ el vector de incógnitas y $\boldsymbol{b}$ el vector de términos independientes. Siendo así, puede ser resuelto eficientemente mediante el Método de Sustitución Regresiva. Es decir:

$$
\begin{gathered}
\lambda_{n} \leftarrow \frac{b_{n}}{a_{w_{n}}} \quad i=n \\
\lambda_{i} \leftarrow \frac{b_{i}-\lambda_{i+1} * a_{e_{i}}}{a_{w_{i}}} \quad i=n-1, \ldots, 1
\end{gathered}
$$

El sistema dado por la ecuación (7), tiene como variable independiente el coeficiente convectivo de transferencia calor (a), parámetro fundamental de la Ley de Enfriamiento de Newton.

En particular, dicho parámetro puede ser obtenido del balance de energía que ingresa $\left(\mathrm{E}_{\text {in }}\right)$ a la madera modelado, el cual es almacenado como energía sensible por la madera húmeda, sumado al calor latente requerido para evaporar el agua extraída. Esto es:

$$
\dot{E}_{\text {in }}=\underbrace{m_{w} c_{p} \frac{d T}{d t}}_{\text {Sensible }}+\underbrace{\dot{m}_{v} h_{f g}}_{\text {Latente }}=\underbrace{\alpha A\left(T_{\infty}-T_{s}\right)}_{\text {Convección }}
$$

Donde $m_{w}$ es la masa de madera húmeda $(\mathrm{kg}) ; c_{p}$ es el calor específico de la madera $(\mathrm{J} / \mathrm{kg} \mathrm{K}) ; d T /$ $d t$ es la tasa de variación de temperatura de la madera $(\mathrm{K} / \mathrm{s}) ; \dot{m}_{v}$ es el flujo de masa de agua evaporada 
de la madera $(\mathrm{kg} / \mathrm{s}) ; h_{f g}$ es el calor latente de vaporización del agua $(\mathrm{J} / \mathrm{kg})$; A es el área de transferencia de calor $\left(\mathrm{m}^{2}\right) ; \alpha$ es el coeficiente convectivo de transferencia de calor $\left(\mathrm{W} / \mathrm{m}^{2} \mathrm{~K}\right) ; T_{\infty}$ es la temperatura del ambiente de secado $(\mathrm{K}) ; T_{s}$ es la temperatura de la superficie de la madera $(\mathrm{K})$.

De lo cual resulta:

$$
\alpha=\frac{m_{w} c_{p} \frac{d T}{d t}+m_{v} h_{f g}}{A\left(T_{\infty}-T_{s}\right)}
$$

\section{RESULTADOS \& DISCUSION}

\section{Curvas de secado y distribuciones de temperatura}

La Figuras $7 \mathrm{a}$ y $7 \mathrm{~b}$ muestran las distribuciones transitorias de $\mathrm{CH}$ y temperatura para el programa $70 / 50^{\circ} \mathrm{C}$. Se puede observar que las probetas de flujo radial y tangencial alcanzaron un CHE de $6,1 \%$ $(80 \mathrm{~h})$ y $8,04 \%$ (96 h), respectivamente. Por otra parte, el incremento de temperatura durante las primeras tres horas, es acelerado hasta superar levemente los $50^{\circ} \mathrm{C}$, donde comienza una ralentización gradual de dicho aumento hasta la temperatura del medio $\left(70^{\circ} \mathrm{C}\right)$. Se observa que, durante el proceso de ralentización se presentan dos eventos que inducen inflexiones relevantes en las variación de temperaturas, los cuales podrían estar asociados: el primero, al $\mathrm{CH}$ Crítico $(\mathrm{CHC})$, ralentización del calentamiento de la madera que se extiende por un par horas (Figura 7a, detalle A) y el segundo a la llegada al PSF, más extenso (5 h), donde es acelerado el calentamiento, (Figura 7a, detalle B). Dicho fenómeno es más intenso y extendido en el tiempo en las probetas de flujo radial versus las probetas de flujo tangencial. En particular, para el secado de flujo radial (Figura 7a), el fenómeno ocurre en torno a $1(\mathrm{~h})$ y $20(\mathrm{~h})$ de haber iniciado el proceso y concluye cuando se alcanzan las proximidades del CHC y PSF (105\% y 30\%), respectivamente. Resultados similares son obtenidos para los programas acelerados, cuyos valores medios, contractados con los de secado convencional, son dados en la Figura $7 \mathrm{c}$ y $7 \mathrm{~d}$, en función de variables dependientes adimensionales $\mathrm{CH}^{*}$ y $\mathrm{T}^{*}$, según definición dada en la descripción de la Figura 7. 


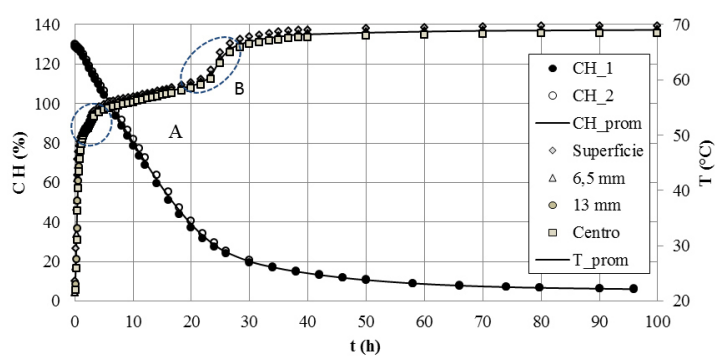

a) $\operatorname{Radial}\left(70 / 50^{\circ} \mathrm{C}\right)$

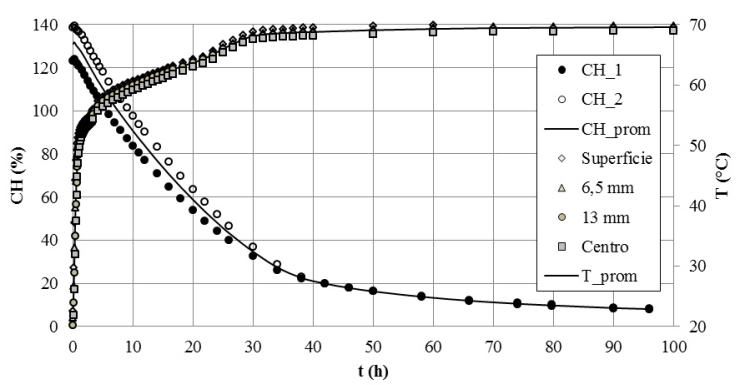

b) Tangencial $\left(70 / 50^{\circ} \mathrm{C}\right)$

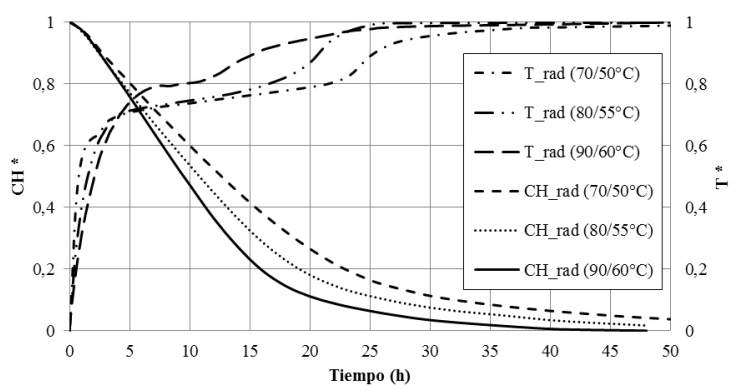

c) Media radial

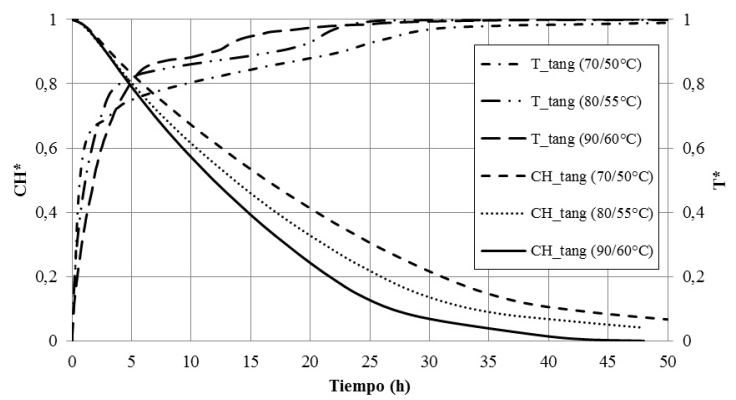

d) Media tangencial

Figura 7. Temperatura y contenido de humedad.

$\left.\left(\mathrm{CH}^{*}=(\mathrm{CH}-\mathrm{CHE}) /\left(\mathrm{CH}_{\mathrm{i}}-\mathrm{CHE}\right)\right), \mathrm{T}^{*}=\left(\mathrm{T}-\mathrm{T}_{\mathrm{i}}\right) /\left(\mathrm{T}_{\mathrm{S}}-\mathrm{T}_{\mathrm{i}}\right)\right)$. 
En este contexto, la bibliografía muestra resultados similares. En particular, Haque (2007) reportó, para el secado acelerado de pino radiata (programa $90 / 60^{\circ} \mathrm{C}$ ) de piezas de pino radiata de similar espesor, una primera fase de incremento acelerado de temperatura hasta $60^{\circ} \mathrm{C}$, que termina en torno de la primera hora de secado, posterior a lo cual, el aumento se ralentiza progresivamente hasta llegar a la temperatura de equilibrio con el ambiente $\left(90^{\circ} \mathrm{C}\right)$. Beard et al. (1983), muestran evidencias similares en cuanto a la aceleración de la variación de temperaturas en la etapa de ralentización, en procesos de secado a alta temperatura (programas $150 / 80$ y $120 / 55^{\circ} \mathrm{C}$ ), lo cual se produjo para un $\mathrm{CH}$ en torno del $15 \%$.

\section{Contenido de humedad}

La Figura 8, muestra la distribución espacial de humedad en la madera para distintos tiempos característicos de la curva de secado. En todas las gráficas es posible observar la pérdida de masa que sufre la madera a medida que aumenta el tiempo de secado. En este contexto, se observa un $\mathrm{CH}$ creciente desde la superficie al centro de la madera. Particularmente, para el programa $70 / 50^{\circ} \mathrm{C}$ (Figura 8a), se aprecia una diferencia de humedad importante entre el centro y la superficie de la madera: $33,79 \%$ a las 8,5 (h) de secado en la probeta de flujo radial. Dicha diferencia disminuye de manera gradual hasta el PSF, a partir de lo cual, la diferencia de humedades es mínima. Asimismo, para el sentido de flujo tangencial (Figura 8b), se tiene una diferencia de humedad máxima a las 11 (h) equivalente a un $31,87 \%$, la cual es minimizada a partir del PSF.

De manera similar, resulta el análisis de los programas $80 / 55^{\circ} \mathrm{C}$ y $90 / 60^{\circ} \mathrm{C}$. No obstante, se evidencian mayores variaciones de humedad entre la superficie y el centro de la madera en los programas acelerados, iguales a $47,11 \%$ y $61,70 \%$ para el programa $80 / 55^{\circ} \mathrm{C}$ y $90 / 60^{\circ} \mathrm{C}$, respectivamente, en probetas de flujo radial (Figura 8c y Figura 8e). En este contexto, Haque (2007) obtuvo resultados similares para el programa $90 / 60^{\circ} \mathrm{C}$, donde la diferencia de humedad en la primera hora de ensayo fue de $90 \%$; y $70 \%$, a las 10 horas de haber iniciado el proceso de secado.

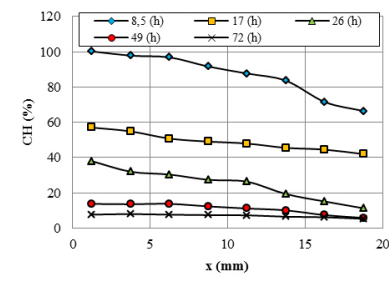

a) Radial $\left(70 / 50^{\circ} \mathrm{C}\right)$

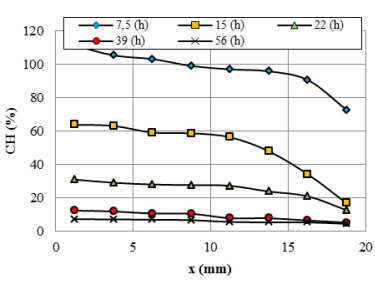

a) $\operatorname{Radial}\left(80 / 55^{\circ} \mathrm{C}\right)$

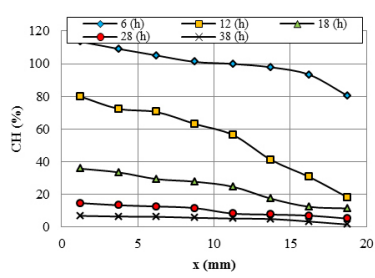

a) Radial $\left(90 / 60^{\circ} \mathrm{C}\right)$

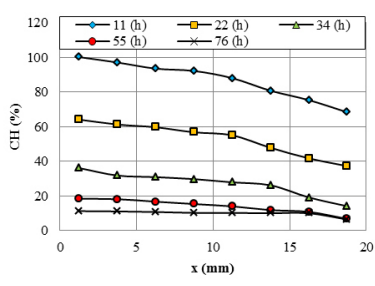

b) Tangencial $\left(70 / 50^{\circ} \mathrm{C}\right)$

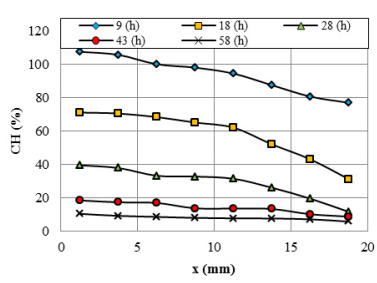

b) Tangencial $\left(80 / 55^{\circ} \mathrm{C}\right)$

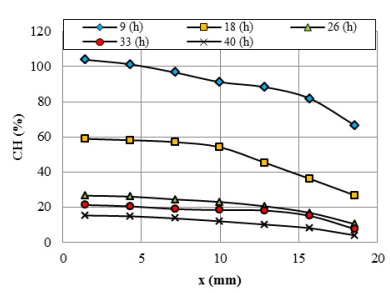

b) Tangencial $\left(90 / 60^{\circ} \mathrm{C}\right)$

Figura 8: Distribuciones espaciales de $\mathrm{CH}$. 


\section{Medición propiedades térmicas}

Los resultados de las mediciones de conductividad térmica y calor específico se muestran en la Figura 9. En particular, la conductividad térmica varía entre 0,18 y $0,60(\mathrm{~W} / \mathrm{m} \mathrm{K})$, conforme el $\mathrm{CH}$ varía entre 2,5 y $158 \%$, respectivamente, lo cual es consistente con los valores obtenidos por Haque (2007), el cual determinó variaciones entre 0,28 y $0,50(\mathrm{~W} / \mathrm{m} \mathrm{K})$, de acuerdo a correlaciones dadas por Pang (1996) y Nijdam et al. (2000). Asimismo, Vay et al. (2015), reportaron que la conductividad fluctúa entre 0,1 y $0,45(\mathrm{~W} / \mathrm{m} \mathrm{K})$ para todas direcciones y sin distinción de especies. En general, se observa que la conductividad térmica presenta tendencias diferentes conforme esté sobre o bajo el 60\% de $\mathrm{CH}$, o dicho de otra manera, conforme esté por sobre o bajo de las proximidades del CHC. Sin duda que los efectos de tener ambientes bifásicos de agua en la madera repercuten en la magnitud de esta propiedad física, tornando compleja su determinación.

Por otro lado, el calor específico experimental oscila entre 2925 y 8500 (J/kg K) para rangos de humedad entre 2,5 y $158 \%$, respectivamente. De manera similar, Haque (2007) evaluó esta propiedad empleando correlaciones dadas por Pang (1996), donde los valores fluctúan entre 1500 y 7500 (J/kg K); en cambio, Nijdam et al. (2000) reporta fluctuaciones entre 1800 y $3400(\mathrm{~J} / \mathrm{kg} \mathrm{K})$, y por último, Haque y Langrish (2001), presentan oscilaciones que van desde 2800 hasta 3500 (J/kg K).

En síntesis, los valores determinados de conductividad térmica y calor específico están dentro de los rangos esperados de acuerdo a la evidencia bibliográfica. Además, según se observa en la Figura 9, la conductividad térmica crece en forma exponencial para $\mathrm{CH}$ desde el estado anhidro hasta las inmediaciones del CHE, a partir de lo cual, se torna lineal su incremento hasta el entorno del CHC. En este entorno $(70 \%)$ se tiene un crecimiento relevante de la conductividad, el cual disminuye conforme se avanza al estado de saturación. Los valores de calor específico presentan un comportamiento singular para valores cercanos al CHE, o más bien, sustantivamente bajo el PSF. Por encima del PSF, la variación del calor específico presenta una variación parabólica. Dichos comportamientos denotan que la propiedad de almacenamiento de energía térmica es una función compleja del contenido de agua ligada. Ciertamente, es esperable que la madera se comporte diferente conforme su nivel de $\mathrm{CH}$ : $\mathrm{CH}$ que permiten conectividad hídrica en estado líquido (sobre $\mathrm{CHC}$ ), $\mathrm{CH}$ dado por mezclas de gas y líquido en las cavidades celulares (entre CHC y PSF) y $\mathrm{CH}$ con presencia de sólo gas en las cavidades celulares proveniente de sus paredes (bajo el PSF).

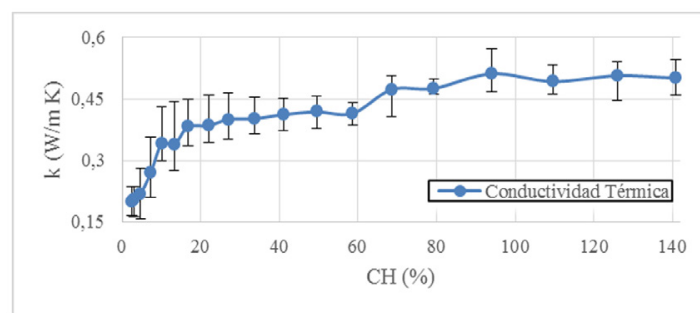

a)

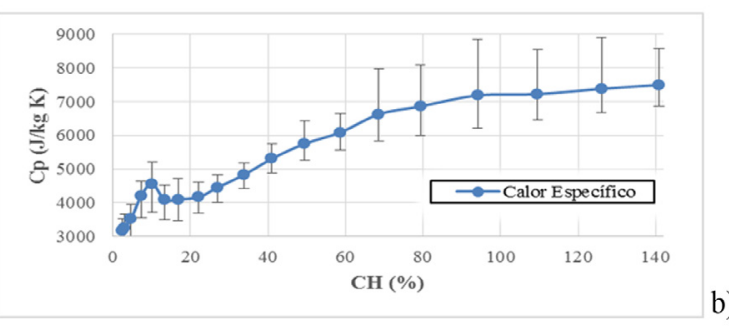

Figura 9: Propiedades térmicas en función de $\mathrm{CH}$ determinadas experimentalmente a través del equipo Decagon KD2-Pro a) conductividad térmica y b) calor específico. 


\section{Determinación inversa de la conductividad}

En la evolución transitoria de temperaturas del proceso $70 / 50^{\circ} \mathrm{C}$ (Figura $7 \mathrm{a}$ ), en el intervalo de tiempo entre 5 y 20 (h), se observa un comportamiento estacionario en el sentido que las temperaturas al interior de la madera evolucionan de manera uniforme como se muestra en detalle en la Figura 10a. Dicha región es particularmente conveniente para determinar parámetros de transporte de calor, no dependientes de las etapas del secado. Siendo así, el mencionado intervalo de distribuciones transitorias de temperaturas, fue utilizado para calcular la conductividad térmica de la madera en función del $\mathrm{CH}$ a través de metodología inversa.

Para calcular el coeficiente convectivo, los valores $\mathrm{T}_{\mathrm{s}}, d T / d t, \mathrm{~m}_{\mathrm{w}} \mathrm{y} \dot{m}_{v}$ requeridos por la ecuación (8), se han obtenido de la distribución de temperaturas y curvas de secado, según corresponda. En cuanto al valor de c, se utilizó el valor experimentalmente, dado en la Figura 9. En particular, para el programa 70/50 $\left({ }^{\circ} \mathrm{C}\right)$, la variación de a en función del $\mathrm{CH}$, requerido por el modelo de problema inverso, es mostrado en la Figura 10b, la cual puede ser aproximada por una función del tipo parabólica.

Las conductividades térmicas obtenidas por metodología inversa para el programa $70 / 50\left({ }^{\circ} \mathrm{C}\right)$, que resultan de la solución del sistema de ecuaciones (ecuación 7), se muestran en la Figura 10c, incluidas, líneas de tendencia y valor determinado experimentalmente a través de equipo Decagon KD2-Pro. Es posible observar una fuerte proporcionalidad entre la conductividad térmica y el $\mathrm{CH}$ de la madera. Dicho comportamiento se debe a que conforme se elimina la humedad de la madera (particularmente agua líquida), se producen cavidades (poros) de vapor y/o aire, los cuales poseen conductividades térmicas menores al agua líquida evacuada. Los resultados medios de conductividad térmica calculada por metodología inversa, para los tres programas de secado analizados $70 / 50,80 / 55$ y 90/60 $\left({ }^{\circ} \mathrm{C}\right)$, son mostrados en la Figura 10d, junto a sus líneas de tendencia. En dicha figura, se puede observar que, además de la humedad, la conductividad térmica depende de la temperatura de la madera.

El fenómeno anterior ha sido discutido por Haque (2007), donde analiza la variabilidad de la conductividad térmica por medio de dos correlaciones obtenidas por Pang (1996) y Nijdam (2000). En este contexto, las correlaciones denotan que la conductividad térmica de la madera depende del $\mathrm{CH}$, de la densidad básica y, también, de la temperatura (Nijdam 2000). Del mismo modo, Zhao et al. (2015) demostraron que la conductividad térmica en madera de alerce europeo (Larix gmelinii Rupr.) dependía igualmente de ambas variables, encontrando fluctuaciones que van desde $0,068(\mathrm{~W} / \mathrm{m} \mathrm{K})$ para temperaturas de $40^{\circ} \mathrm{C}$ y $0 \%$ de humedad, hasta $0,151(\mathrm{~W} / \mathrm{m} \mathrm{K})$ para temperaturas de $70^{\circ} \mathrm{C} \mathrm{y} 80 \%$ de humedad. Si bien la temperatura influye sobre la magnitud de la conductividad térmica, es el $\mathrm{CH}$ el que más incide sobre esta propiedad, lo que es corroborado por el presente estudio. Particularmente, en el estudio de Zhao et al. (2015), se alcanzaron diferencias de 18,89\% entre la temperatura mínima y máxima analizadas $\left(40\right.$ y $70^{\circ} \mathrm{C}$, respectivamente) para un $\mathrm{CH}$ máximo $(80 \%)$; y $91,13 \%$ para una temperatura de $70^{\circ} \mathrm{C}$ cuando la variación de humedades fluctúa entre $0 \%$ hasta $80 \%$.

Entonces, la tendencia de la conductividad térmica calculada por medio de la metodología inversa para los tres programas de secado cumple con el planteamiento anterior, es decir, las diferencias porcentuales aumentan conforme aumenta el $\mathrm{CH}$ de la madera y la temperatura, cuya diferencia máxima se obtiene entre los límites de temperatura estudiados: $70^{\circ} \mathrm{C} \mathrm{y} 90^{\circ} \mathrm{C}$; alcanzando un valor medio de $9,87 \%$ con una desviación estándar de 2,36\%. Cuando se comparan los valores calculados por metodología inversa versus los experimentales (Decagon KD2-Pro), los resultados asociados al programa comparable, $70 / 50\left({ }^{\circ} \mathrm{C}\right)$ presentan una diferencia máxima de $(12,70 \%)$, con una desviación estándar de 5,92\%. 


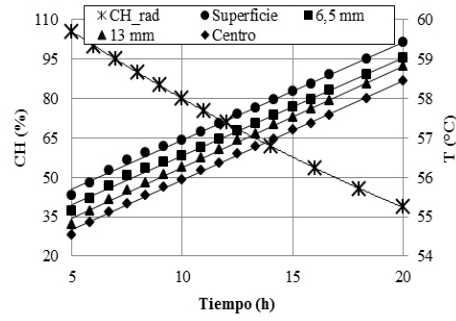

a) Temperatura $\left(70 / 50^{\circ} \mathrm{C}\right)$

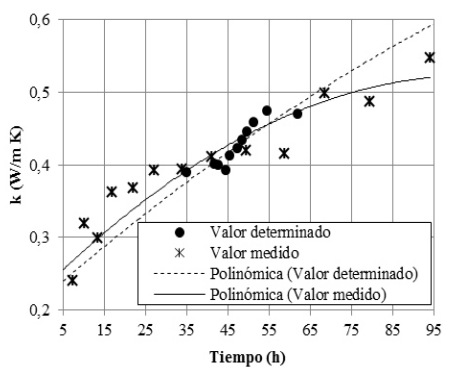

c) Conductividad térmica $\left(70 / 50^{\circ} \mathrm{C}\right)$

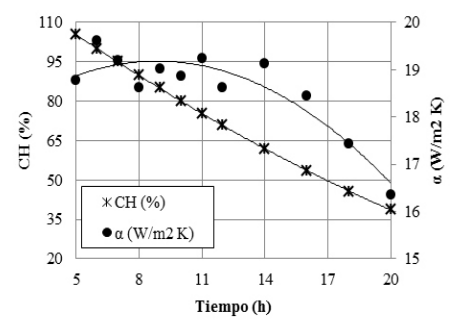

b) Coeficiente convectivo $\left(70 / 50^{\circ} \mathrm{C}\right)$

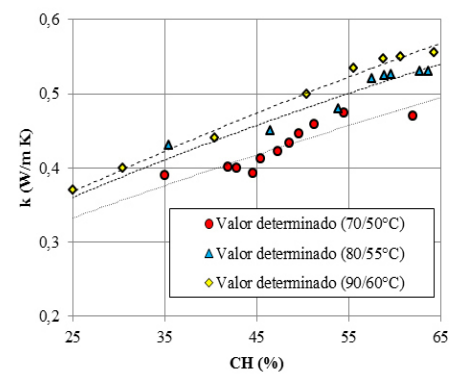

d) Conductividad media

Figura 10: Determinación inversa de conductividad.

\section{CONCLUSIONES}

Se obtuvieron datos experimentales de distribuciones transitorias y espaciales del $\mathrm{CH}$ y temperaturas, para tres programas de secado de pino radiata, los cuales sirvieron al propósito de la determinación inversa de la conductividad térmica en función del $\mathrm{CH}$.

En particular, las curvas de temperatura entregaron información relevante acerca del comportamiento de la madera en el proceso, lográndose identificar regiones con aceleraciones y desaceleraciones de incrementos de temperatura. Dichas regiones responden a alteraciones de la madera húmeda debido a la disminución de su CH durante el proceso de secado, situándose en las cercanías de puntos característicos de la curva de secado: el CHC y el PSF.

Para la implementación de la metodología inversa, se seleccionó una región del proceso caracterizado por un comportamiento pseudo lineal de la distribución de temperatura. En este contexto, se determinó satisfactoriamente la conductividad térmica de la madera en función del $\mathrm{CH}$, cuyos resultados presentaron tendencias acorde a datos disponibles en la literatura especializada. Asimismo, la metodología inversa fue capaz de captar la dependencia de la conductividad térmica con la temperatura: proporcionalidad entre conductividad térmica y temperatura.

Por último, la determinación de conductividad y calor específico usando el instrumento KD2-Pro, sirvió, efectivamente, al doble propósito de validar resultados calculados de conductividad a través de metodología inversa y complementar las propiedades física requeridas por el modelo matemático.

\section{AGRADECIMIENTOS}

Los autores agradecen el financiamiento de este trabajo al proyecto Conicyt, Fondef Idea 2-Etapas ID14I10231. 


\section{REFERENCIAS}

Ananías, R. A.; Vallejos, S.; Salinas, C. 2005. Estudio de la Cinética del Secado Convencional y Bajo Vacío del Pino Radiata. Maderas-Cienc Tecnol 7(1): 37-47.

Ananías, R.A.; Lastra, J.; Salvo, L.; Contreras, H.; Barría, C.; Peredo, M. 2010. Estudio exploratorio de la variabilidad radial y apical del tamaño y frecuencia de los canales resiníferos en pino radiata. Maderas-Cienc Tecnol 12(2): 135-142.

Beard, J.; Rosen, H.; Adesanya, B. 1983. Temperature, Distributions and Heat Transfer During the Drying of Lumber. Drying Technology 1(1): 117-140.

Cai, L. 2005. Determination of Diffusion Coefficients for Sub-alpine fir. Wood Science Technology 39: $153-162$.

Cai, L.; Garrahan, P. 2006. Inverse Determination of Thermal Conductivity in Frozen Lumber. Wood Science Technology 40: 665-672.

Czajkowski, L.; Olek, W.; Weres, J.; Guzenda, R. 2016. Thermal Properties of Wood-Based Panels: Thermal Conductivity Identification with Inverse Modeling. Eur J Wood Prod 74: 577-584.

Derdour, L.; Desmorieux, H.; Andrieu, J. 2007. The Critical Moisture Content (c.m.c.) and the Internal Moisture Content Profile During the Constant Drying rate Period. Drying Technology $16(3), 813-824$.

Diaz-vaz; J.E.; Fernandez, A.; Valenzuela L.; Torres, M. 2007. Madera de compresión en Pinus radiata: I Características anatómicas. Maderas-Cienc Tecnol 9(1):29-43.

Diaz-vaz, J.E.; Ananías, R.A.; Rodriguez, S.; Torres, M.; Fernández, A.; Valenzuela, L. 2009. Compression wood in Pinus radiata II: Density and chemical composition. Maderas-Cienc Tecnol 11(2):139-151.

Haque, M.; Langrish, T. 2001. Stack-wide Effects in the Modeling of Solar Kilns for Drying Timber. Drying Technology 19(1): 99-114.

Haque, M. 2007. Simulation of Temperature and Moisture Content Profiles in a Pinus radiata Board During High-Temperature Drying. Drying Technology 25: 547-555.

INFOR 2016. La Industria del Aserrío. Boletín Estadístico Nº 155. Instituto Forestal, SantiagoChile.

Instituto Nacional de Normalización. NCh. 1986. Madera - Selección, Obtención y Acondicionamiento de Muestras y Probetas para la Determinación de Propiedades Físicas y Mecánicas. NCh968.Of86. Santiago, Chile, 1986.

Liu, J.; Simpson, W.; Verrill, S. 2001. An Inverse Moisture Diffusion Algorithm for the Determination of Diffusion Coefficient. Drying Technology 19(8): 1555-1568.

Luikov, A. V. 1966. Heat and mass transfer in capillary porous bodies. Pergamon Press, Oxford. pp 522.

Nijdam, J.; Langrish, T.; Keey, R. 2000. A High-Temperature Drying Model for Softwood Timber. Chemical Engineering Science 55: 3585-3598.

Özisik, M.; Orlande, H. 2000. Inverse Heat Transfer. Taylor \& Francis. 330pp.

Pang, S. 1996. Moisture Content Gradient in a Softwood Board Drying: Simulation From a 2-D Model and Measurement. Wood Science and Technology 30: 165-178.

Patankar, S. V. 1980. Numerical Heat Transfer and Fluid Flow. Hemisphere Publishing Corporation, N. Y. 197pp.

Pérez-Peña, N.; Valenzuela, L.; Díaz-Vaz, J.E.; Ananías, R.A. 2011. Predicción del contenido de humedad de equilibrio de la madera en función del peso específico de la pared celular y variables ambientales. Maderas-Cienc Tecnol 13(3):253-266. 
Salinas, C.; Ananías, R.A.; Ruminot, P. 2008. Modelación fenomenológica de las curvas de secado por alta temperatura de pino radiata. Maderas-Cienc Tecnol 10(3): 207-217.

Salinas, C.; Chávez, C.; Ananías, R.A.; Elustondo, D. 2015. Unidimensional simulation of drying stresses in radiata pine wood. Drying Technology 33 (8):996-1005.

Vay, O.; De Borst, K.; Hansmann, C.; Teischinger, A.; Muller, U. 2015. Thermal conductivity of wood at angles to the principal anatomical directions. Wood Sci Technol 49(3): 577-589.

Versteeg, H.K.; Malalasekera, W. 1995. An Introduction to Computational Fluid Dynamics. Longman Scientific \& Technical. 257p.

Zhao, J.; Fu, Z.; Jia, X.; Cai, Y. 2015. Inverse Determination of Thermal Conductivity in Lumber based on Genetic Algorithms. Holzforschung 70: 1-7.

Whittaker, S. 1977. Simultaneous heat, mass, and momentum transfer in porous media: A theory of drying. Advances in Heat Transfer 13: 119- 203.

Zhang, J.; Miao, P.; Zhong, D.; Liu, L. 2014. Mathematical modeling of drying of Masson pine lumber and its asymmetrical moisture content profile. Holzforschung 68(3): 313-321.

Zhao, J.; Fu, Z.; Jia, X.; Cai, Y. 2016. Modeling Conventional Drying of Wood: Inclusion of a moving evaporation Interface. Drying Technology 34(5): 530-538.

Zheng, S.; Song, K.; Zhao, J.; Dong, C. 2016. Inverse Estimation of Effective Moisture Diffusivity in Lumber During Drying Using Genetic Algorithms. BioResources 11(4): 8226-8238. 